Thorax (1961), 16, 94.

\title{
CONGENITAL TRACHEAL STENOSIS
}

\author{
BY \\ I. B. HOUSTON* AND D. G. MACKIE \\ From the Hospital for Sick Children, Great Ormond Street, London
}

(RECEIVED FOR PUBLICATION OCTOBER 6, 1960)

Congenital stenosis of the trachea, though a rare disease, has been recognized for many years, but it is not generally realized that it can be a cause of stridor in infancy. We feel, therefore, that it is worth reporting a patient with this malformation, especially as a limited number of these patients may have the condition corrected by surgery.

\section{Case Report}

A boy, aged 8 months, was admitted to the Hospital for Sick Children, Great Ormond Street, under the care of Dr. B. E. Schlesinger, on May 27, 1959. He weighed $6 \mathrm{lb}$. $4 \mathrm{oz}$. at birth and required oxygen therapy for about five minutes before adequate breathing was established. Noisy respiration was noted at once, but gradually subsided in the next few days, the stridor becoming barely discernible. The stridor became temporarily worse following a right inguinal herniorrhaphy at the age of 3 months and much worse at the ages of 4 months and 7 months, on each of these occasions being associated with cough, dyspnoea, cyanosis, and pyrexia. These attacks, presumably due to respiratory infection, were much improved by antibiotic and oxygen therapy, but from the age of 4 months he experienced attacks of apnoea and cyanosis, each lasting about a minute, from which he recovered spontaneously. His general health and development were surprisingly good, but dyspnoea and sometimes cyanosis were easily provoked by feeding or any form of exercise ; no dysphagia was noted.

His two elder brothers, aged $3 \frac{1}{2}$ and 5 years, his parents and grandparents were well, and no history of maternal illness or of exposure to $x$ rays while pregnant could be elicited.

On examination he was dyspnoeic with inspiratory and expiratory stridor and indrawing of the supraclavicular tissues on inspiration. The only other abnormalities detected on full examination were a soft systolic bruit, best heard at the left sternal border, and a few rales scattered through the chest.

His haemoglobin was $10.4 \mathrm{~g} . / 100 \mathrm{ml}$., the white cell count $5,500 / \mathrm{c} . \mathrm{mm}$., with a normal differential count, and the Mantoux test, using a 1:1,000 dilution of old tuberculin, was negative. A radiograph of the chest showed an area of consolidation in the right

\footnotetext{
*Present address: Royal Manchester Children's Hospital, Pendlebury, near Manchester, Lancs.
}

middle lobe which cleared, radiologically, following antibiotic therapy. Barium swallow did not show a vascular ring constricting the trachea or oesophagus.

A laryngoscopy, performed by Mr. James Crooks, revealed no abnormality, but it was impossible to pass a bronchoscope right down the trachea. In consequence bronchography was performed and posteroanterior and lateral views of the resulting bronchogram are shown in Fig. 1. It showed a gradual constriction of the distal three-quarters of the trachea, maximal at the bifurcation, where the main bronchi branched virtually at right angles.

A diagnosis of tracheal stenosis was made, which, by virtue of its length, was considered inoperable and the patient was discharged from the hospital taking oral phenoxymethylpenicillin, $125 \mathrm{mg}$. twice daily, to try to prevent further respiratory infections and minimize the tracheal obstruction. Unfortunately, a few weeks later, after two days' apparently mild respiratory infection, he developed acute respiratory obstruction, was admitted to hospital (in Malta), and died.

Post-mortem examination by Major W. E. Clifford, R.A.M.C., showed that the trachea was increasingly constricted as it descended, with extreme narrowing at its bifurcation. The tracheal rings were circular and continuous, rather than the usual horseshoe shape, and the mucosa of the larynx, trachea, and bronchi was acutely inflamed and oedematous, thus accentuating the tracheal stenosis. There were numerous petechiae on the pleural surface of the lungs and a little straw-coloured fluid was found in both pleural and pericardial sacs. No other abnormalities were found except an atrial septal defect.

\section{Discussion}

Wolman (1941) reported a case of tracheal stenosis presenting in a girl of 2 months of age, and was able to find 10 other cases recorded in the literature. He classified these into two groups, one (type A), where the stenosed segment was short and might be produced by only one or two deformed tracheal cartilages, and the other (type B) where the stenosis involved the larger part of the trachea and sometimes the main bronchi (Fig. 2). The second type was due to the absence of the membranous (posterior) part of the trachea, so that the tracheal cartilages were complete rings 


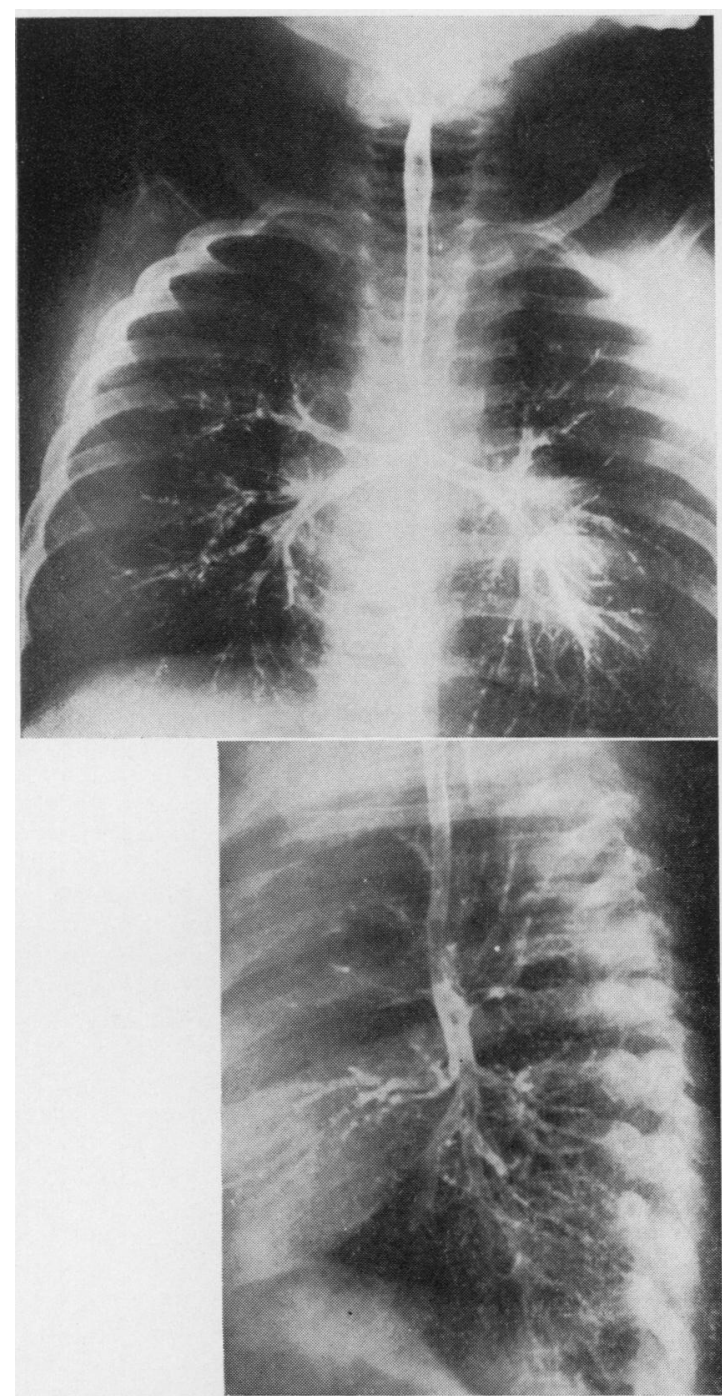

Fig. 1.-Postero-anterior and lateral views of bronchogram.

encircling and constricting it. This constriction was usually maximal just above the bifurcation and, as a result, the trachea assumed a funnel shape. Heikel (1957) collected more cases from the literature and increased the list to 22 patients. $\mathrm{He}$ also added a further example of Wolman's type B stenosis.

Many other congenital anomalies have been found in association with tracheal stenosis ranging from clubfoot and congenital absence of the thumbs (Wolman, 1941) to imperforate anus (Bonnier, 1947). Congenital heart disease has been associated several times, including ventricular septal defect (Montandon, 1944), persistent truncus arteriosus (Heikel, 1957), and atrial septal defect (the present case). The congenital anomalies most closely associated, however, are those found in the lungs; indeed, it is the rule, rather than the exception, for cases of tracheal stenosis to be associated with some pulmonary malformation. This has varied from merely anomalous lobulation (Weber, 1952) to areas of atalectasis (Wolman, 1941) and even aplasia of the lung (Putney and Baltzell, 1952).
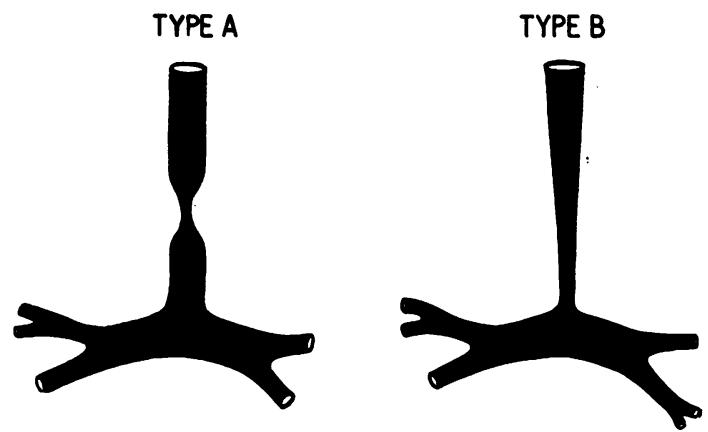

FIG. 2.-Wolman's classification of congenital tracheal stenosis.

The prognosis of untreated patients is difficult to assess. The majority of reported cases have died in infancy, but a few have survived on conservative treatment, and, if the patient can be sustained through the first few years of life, the disability may gradually decrease with the increasing size of the trachea. Some patients with minor degrees of stenosis remain asymptomatic or have so little stridor that they are not recognized as instances of this malformation. That this occurs is shown by the report of Stewart and Pinkerton (1955) of a 60-year-old patient in whom the stenosis (thought to be congenital) was accidentally discovered during the course of a general anaesthetic when an endotracheal tube could not be passed.

Nevertheless, tracheal stenosis sufficiently marked to cause stridor is a serious disease with a high mortality rate, and as some cases are open to surgical correction every effort must be made to locate and assess the narrowing. Review of the literature does not indicate any characteristic clinical features, though the presence of stridor both in inspiration and expiration, especially if it is made worse by respiratory infections, may be suggestive. Plain postero-anterior and lateral radiographs of the chest, which demonstrate the air-filled trachea, may be diagnostic, but care must be exercised to obtain films in inspiration and expiration, because the trachea and bronchial tubes dilate on inspiration and collapse during 
expiration, especially in infants. To confirm the diagnosis it is usually necessary to perform a tracheoscopy or bronchography, and though the latter carries a risk of completely obstructing the narrowed trachea, this risk can be minimized by using a contrast medium in aqueous solution under a general anaesthetic. so that the contrast medium can be aspirated immediately should blockage occur. Moreover, the information so obtained is valuable in differentiating the short type A stenosis from the long type B stenosis and enables a rational approach to therapy to be made.

The type A stenosis can be surgically treated by dilatation, which may need to be repeated, or, if this is inadequate, it may be possible to deal with it in a similar way to that described by Thomson and Negus (1948) for the treatment of laryngeal stenosis, though we know of no case where this has been done. This would involve incising the trachea at the site of the stenosis, dilating it, and repairing the defect with a skin graft held in position by a plastic tube. Where surgical correction is impossible, as in the present case, respiratory infections should be prevented if possible and promptly treated if they do occur. In most cases, prophylactic antibiotic therapy, preferably with one of the tetracycline group, is justifiable, and prolonged close supervision, possibly in hospital, is obviously desirable. Acute exacerbation of the stridor due to a respiratory infection is an indication for urgent antibiotic therapy; humidification of the atmosphere and the use of a mixture of $50 \%$ oxygen and $50 \%$ helium for respiration have been advocated (Wolman, 1941). Tracheotomy cannot be beneficial, though it has, on occasion, been the procedure which finally led to the correct diagnosis.

\section{SUMMARY}

A fatal case of congenital tracheal stenosis is described and the diagnosis, treatment, and prognosis of the condition briefly reviewed.

The majority of reported cases have ended fatally, and we cannot emphasize too strongly the need for prolonged careful supervision to ensure the best possible chance of survival.

We express our thanks to Dr. B. E. Schlesinger and Mr. James Crooks for their kind permission and help in the publishing of this case record, and to Major W. E. Clifford, R.A.M.C., for supplying us with the details of the necropsy findings.

\section{REFERENCES}

Bonnier, M. (1947). Ann. méd.-chir. Hôp. S. Justine Montréal (No. 2), 5, 13

Heikel, P. E. (1957). Ann. Paediat. Fenn., 3, 22

Montandon, A. (1944). Pract. oto-rhino-laryng. (Basel), 6, 179. Montandon, A. (1944). Pract. oto-rhino-laryng. (Basel), 6. 179. 677.

Stewart, S., and Pinkerton, H. H. (1955). Brit. J. Anaesth. . 27, 492 Thomson. S., and Negus, V. E. (1948). Diseases of the Nose and Throat, 5th ed., pp. 704-706. Cassell, London.

Weber, H. W. (1952). Zbl. allg. Path. paih. Anat., 89, 148.

Wolman, I. J. (1941). Amer. J. Dis. Child., 61, 1263. 